Journal of Hospital Infection Volume 62, Issue 2 , February 2006, Pages 181-186

doi:10.1016/j.jhin.2005.08.010

Copyright (c) 2005 The Hospital Infection Society Published by Elsevier Ltd.

\title{
Correlation between the genetic diversity of nosocomial pathogens and their survival time in intensive care units
}

\author{
P. Gastmeier ${ }^{a, *}$, F. Schwab ${ }^{b}$, S. Bärwolff ${ }^{b}$, H. Rüden ${ }^{b}$ and H. Grundmann ${ }^{c}$ \\ ${ }^{a}$ Hannover Medical School, Institute of Medical Microbiology and Hospital \\ Epidemiology, Hannover, Germany \\ ${ }^{b}$ Institute of Hygiene and Environmental Medicine, Charité University Medicine \\ Berlin, Berlin, Germany \\ 'EARSS Project, RIVM, Bilthoven, The Netherlands \\ * Corresponding author. Address: Institute of Medical Microbiology and Hospital \\ Epidemiology, Medical School Hannover, Carl-Neuberg-Str. 1, D 30625 Hannover, \\ Germany. Tel.: +49511532 5147; fax: +495115328174.
}

\section{Summary}

Bacteria differ in their ability to survive in the hospital environment outside the human host. Species remaining viable and infectious have a higher chance of being transmitted, giving them a fitness advantage in hospitals. This differential fitness could be expected to alter the genetic population structure of bacterial populations in hospitals, and should be reflected by the relative abundance of several successful clones. The objective of this study was to test for a potential correlation between tenacity, i.e. environmental survival, and clonal abundance determined by the genetic diversity in different bacterial species from prospectively collected isolates of intensive care patients. A literature review was performed to identify mean environmental survival times for the most important pathogens in intensive care units (ICUs): Staphylococcus aureus, enterococci, Acetinobacter baumannii, Pseudomonas aeruginosa, Enterobacter spp., Escherichia coli, Klebsiella pneumoniae and Stenotrophomonas maltophilia. To determine the genetic diversity of the natural population of these species in ICUs, a prospective 18-month study was conducted in five units with median nosocomial infection rates. All clinical isolates were collected, and highly discriminatory DNA fingerprinting techniques were used to identify specific clones. A diversity index for each species was calculated as the number of distinguishable genotypes in the population divided by size. The correlation between survival times and the diversity indices for the individual pathogens was investigated using non-parametric methods. Although 21 studies were identified in the literature, only two were relevant. They showed median survival times between 1.5 days ( $P$. aeruginosa) and 60.0 days (Enterococcus faecium). During the prospective ICU study, 1264 pathogens were investigated and simple diversity indices between 49.1 (Enterococcus faecalis) and 89.8 (E. coli) were found. A correlation between survival times and the diversity indices for the individual pathogens was found (correlation coefficient 0.821, $P=0.024$ ). Environmental survival may be an important factor contributing to the ecological fitness of some nosocomial pathogens in ICUs. Infection control measures should consider this finding. 


\section{Introduction}

Healthcare-associated infections are a particular threat to patients who are immunocompromised and need intensive care. As treatment becomes limited due to the relentless increase in antibiotic resistance, preventive measures are gaining importance. Most healthcare-associated infections are endogenous, but exogenous infections might be prevented by appropriate infection control procedures.

Pathogens originating from patients or environmental reservoirs are mainly transmitted by hands of personnel or other vectors (such as fomites or devices). Where transmission is vector-borne, bacteria have to survive outside the host milieu that normally supports their survival and replication.

However, the role of the inanimate hospital environment in the transmission of nosocomial infections is controversial. ${ }^{1,2,3,4}$ and 5 Limited data are available on the survival of nosocomial pathogens outside the human host, and the influence of their tenacity on their success in healthcare institutions has never been studied. Therefore, the objective of this study was to see whether the frequency with which certain strains were isolated from intensive care patients is associated with environmental survival time, using the most important nosocomial microorganisms in intensive care untis (ICUs) described in the literature.

\section{Methods}

The investigation focused on 10 of the most frequent nosocomial pathogens in ICUs: Acetinobacter baumannii complex, Enterobacter aerogenes, Enterobacter cloacae, Escherichia coli, Enterococcus faecium, Enterococcus faecalis, Klebsiella pneumoniae, Pseudomonas aeruginosa, Staphylococcus aureus and Stenotrophomonas maltophilia. A MEDLINE search was performed for the terms 'survival' and 'tenacity' using the Boolean operator 'AND' for each species for the period from 1966 until 2004. Only original articles were considered. The articles were scanned for information on survival time, and the methods used were evaluated critically in order to identify studies sufficiently comparable for the different pathogens under investigation. Furthermore, the inocula and test surfaces used had to have been relevant to the ICU environment in order for the study to be included.

\section{Diversity of pathogens}

During an 18-month period (February 2000-July 2001), patients who stayed $\geq 48 \mathrm{~h}$ on five ICUs at two university hospitals were enrolled. The ICUs were encouraged to maintain routine microbiological investigations, and all collected microbiological specimens on clinical grounds at high frequency. All clinical and screening isolates of the 10 'indicator organisms' were collected and stored at $-80^{\circ} \mathrm{C}$ (Microbank, Viva). Only primary isolates from each patient were submitted for molecular typing [S. aureus and enterococci by pulsed-field gel electrophoresis (PFGE); Gram-negative pathogens by AP-polymerase chain reaction technique and a double primer amplified fragment-length polymorphism (AFLP) method]. After typing, genetically indistinguishable organisms isolated from different sites of the same patient were excluded. Full details of the methods used have been published elsewhere. ${ }^{6}$

To describe the genetic population structure for each bacterial species, a simple diversity index was used, as follows. In a population of $N$ isolates of a species in a defined environment, a given isolate will belong to one of $D$ 
distinguishable/different (geno) types. That is why the simple diversity (SD) for a species can be computed as $S D=D / N^{*} 100 . S D$ is a simple measure for the diversity (ecological variety) of a species in the examined population. The $95 \%$ confidence intervals (Exact binomial) were also computed.

\section{Correlation}

To assess the correlation between survival time and simple diversity, a nonparametric correlation analysis was used. Spearman's rank correlation coefficient was calculated as a measure of the strength of the association between survival time and simple diversity, because the sample size was small and the different distribution of the data (normal vs binominal) makes Pearson's correlation coefficient (parametric correlation) undesirable and misleading. SPSS version 11.5.1 and EpiInfo version 6.04d were used for analysis.

\section{Results}

\section{Survival time}

Twenty-one articles on the survival of nosocomial pathogens were identified. Due to methodological differences, the results of most of these studies were not comparable. Wide variations existed between materials contaminated, inocula, incubation conditions (temperature, humidity), and duration of follow-up. Furthermore, most studies did not include sufficient strains. No study investigated all 10 pathogens of interest; however, Neely ${ }^{7}$ and Neely and Maley ${ }^{8}$ reported the survival times for Gram-positive and Gram-negative pathogens using similar methods. Their results were deemed satisfactory for our purposes.

In Neely and Maley's study, the survival of 30 S. aureus strains, 20 E. faecalis strains and 20 E. faecium strains was investigated on five common hospital materials (cotton, terry, blend, polyester and polyethylene) using inocula of $10^{4}-$ $10^{5}$ colony-forming units. Survival was assayed daily by placing the swatches in nutritive media and examining them for growth after $48 \mathrm{~h} .{ }^{8}$ All strains were tested for at least 90 days. In the study by Neely, $10 \mathrm{P}$. aeruginosa strains, $10 \mathrm{E}$. coli strains, $10 \mathrm{~K}$. pneumoniae strains, 10 Acinetobacter spp. strains and 10 Enterobacter spp. strains were studied using the same materials, similar inocula and observation periods of at least 60 days. ${ }^{7}$ Table I summarizes both studies considering only the data of methicillin-sensitive $S$. aureus and vancomycinsensitive enterococci, and the results until day 60 for Gram-positive microorganisms and for the same materials for Gram-negative micro-organisms. The studies showed a large variation in survival between individual strains of the same species, as well as between the various species. The longest median survival time was found for enterococci, followed by Enterobacter spp., S. aureus and Acinetobacter spp. The shortest median survival time under dry conditions was identified for $P$. aeruginosa. Due to the lack of data for $S$. maltophilia, this was excluded from the present study; $E$. cloacae and $E$. aerogenes were combined because survival data were only available for Enterobacter spp. 
Table I.

Survival time data for various pathogens and materials according to the studies of Neely ${ }^{7}$ and Neely and Maley ${ }^{8}$

\begin{tabular}{|l|l|l|l|l|l|}
\hline Pathogen & $\begin{array}{c}\text { Strains } \\
\text { investigated and } \\
\text { considered for this } \\
\text { analysis }\end{array}$ & \multicolumn{4}{|l|}{ Survival time (days) } \\
\hline & \multicolumn{1}{|c|}{$\begin{array}{l}\text { Minimum } \\
\text { Maximum }\end{array}$} & \multicolumn{1}{|c|}{ Median } & Median \\
\hline Staphylococcus aureus (MSSA) & 15 & 1 & $>60$ & 12 & 12.0 \\
\hline Enterococcus faecium (VSE) & 10 & 22 & $>60$ & $>60$ & 60.0 \\
\hline E. faecalis (VSE) & 10 & 11 & $>60$ & 31 & 31.0 \\
\hline Enterobacter spp. & 10 & 5 & 49 & 17.5 & 17.5 \\
\hline Klebsiella pneumoniae & 10 & 4 & 27 & 7.5 & 7.5 \\
\hline Escherichia coli & 10 & $13 \mathrm{~h}$ & 25 & 2 & 2.0 \\
\hline Acinetobacter spp. & 10 & 1 & $>60$ & 9 & 9.0 \\
\hline Pseudomonas aeruginosa & 10 & $2 \mathrm{~h}$ & 10 & 1.5 & 1.5 \\
\hline
\end{tabular}

VSE, vancomycin-sensitive enterococci; MSSA, methicillin-sensitive S. aureus.

\section{Diversity of pathogens}

Between February 2000 and July 2001, 7269 admissions totalling 35817 patientdays were recorded in the five study ICUs. For 1876 admissions $(25.8 \%)$, treatment intervals lasted $\geq 48 \mathrm{~h}$ (28 498 patient-days). The microbiology laboratory received 30033 samples (1.05/patient/day). These yielded 4953 pathogens, of which 2733 were of the indicator strains. After eliminating duplicates, 1264 isolates were submitted to molecular typing.

Table II shows the results for those micro-organisms together with their survival time data taken from the studies by Neely ${ }^{7}$ and Neely and Maley. ${ }^{8}$ The genetic diversity for the pathogens included is very heterogenous; for $E$. faecium and $E$. faecalis, it is significantly lower than the mean diversity index, whereas the diversity for E. coli, K. pneumonia and Enterobacter spp. is significantly higher. The confidence intervals for $S$. aureus and Acinetobacter spp. include the mean diversity index.

Table II.

Survival time and diversity of micro-organisms

\begin{tabular}{|l|l|l|l|l|l|}
\hline Micro-organisms & $\begin{array}{c}\text { Median } \\
\text { survival } \\
\text { time } \\
\text { (days) }\end{array}$ & $\begin{array}{c}\text { Number } \\
\text { of } \\
\text { isolates }\end{array}$ & $\begin{array}{c}\text { Number of } \\
\text { indistinguishable } \\
\text { genotypes }\end{array}$ & $\begin{array}{c}\text { Simple } \\
\text { diversity } \\
\text { index }\end{array}$ & $\mathbf{9 5 \%}$ CI \\
\hline Staphylococcus aureus & 12.0 & 456 & 340 & 74.6 & $70.3-78.5$ \\
\hline Enterococcus faecium & 60.0 & 60 & 37 & 61.7 & $48.2-73.9$ \\
\hline Enterococcus faecalis & 31.0 & 169 & 83 & 49.1 & $41.6-57.2$ \\
\hline Enterobacter spp. & 17.5 & 105 & 90 & 85.7 & $77.5-91.8$ \\
\hline Klebsiella pneumoniae & 7.5 & 80 & 69 & 86.2 & $76.7-92.9$ \\
\hline Escherichia coli & 2.0 & 157 & 141 & 89.8 & $84.0-94.1$ \\
\hline Acinetobacter spp. & 9.0 & 30 & 21 & 70.0 & $50.5-91.8$ \\
\hline Pseudomonas aeruginosa & 1.5 & 134 & 111 & 82.8 & $75.4-88.8$ \\
\hline Total & & 1191 & 892 & 77.9 & \\
\hline
\end{tabular}

${ }^{\text {a }}$ According to Table I.

${ }^{\mathrm{b}}$ Without duplicates. 


\section{Association of survival time and simple diversity}

The association between survival time under dry conditions and simple diversity is shown in Figure 1 . The Spearman rank correlation coefficient was 0.821 , which means a significant correlation $(P=0.024)$.

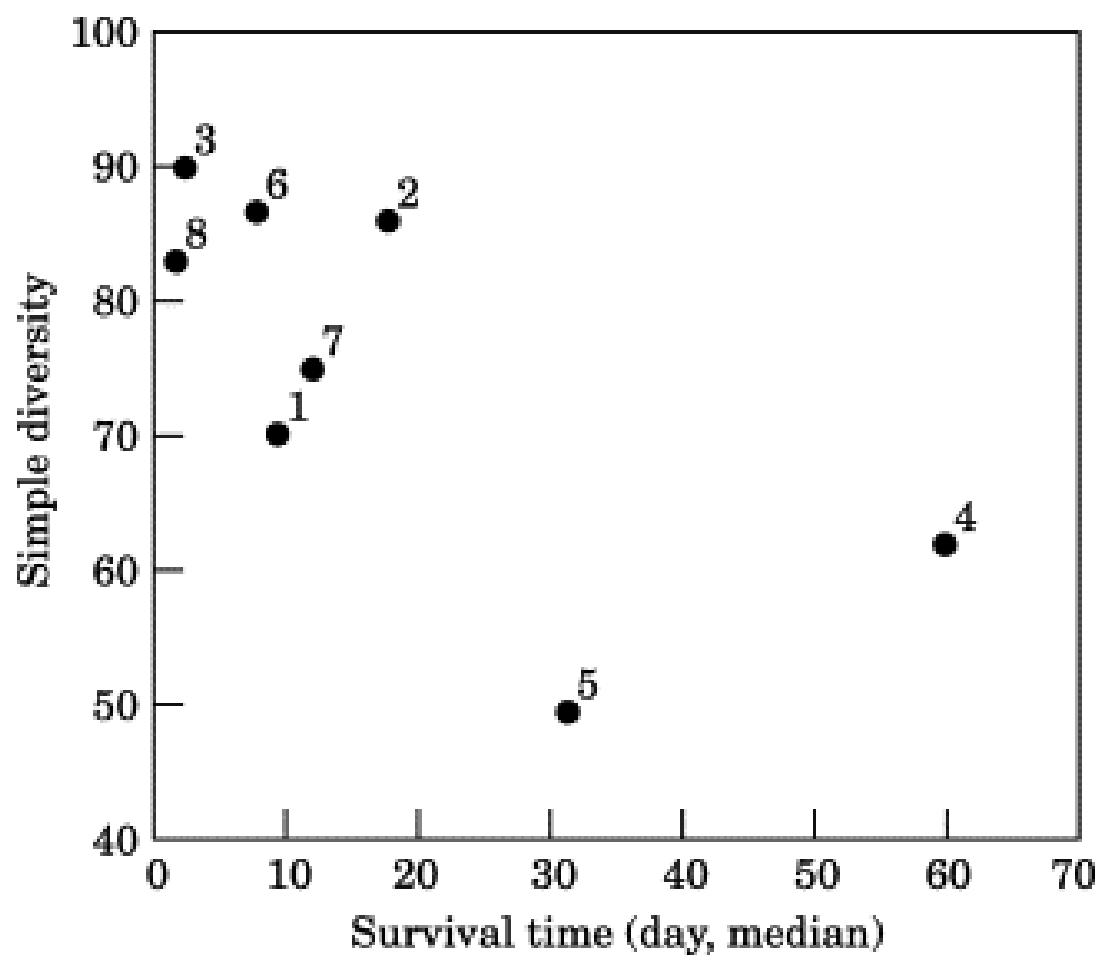

Figure 1. Association between survival time and simple diversity. 1, Acinetobacter spp.; 2, Enterobacter spp.; 3, Escherichia coli; 4, Enterococcus faecium; 5, Enterococcus faecalis; 6, Klebsiella pneumoniae; 7, Staphylococcus aureus; 8 , Pseudomonas aeruginosa.

\section{Discussion}

This study demonstrates an intriguing degree of correlation between survival under ambient conditions in ICUs and the genetic diversity of the most important bacterial pathogens that cause nosocomial infections in these settings. It is the authors' hope that this finding will stimulate discussion about the potential role of environmental contamination in healthcare-associated infections. Factors that may curtail the validity of these observations are discussed below.

\section{Sampling bias}

With the exception of $S$. aureus (diagnostic and screening samples), most isolates came from diagnostic samples. This may have over-represented the proportion of pathogens to colonizers. For most species, it is unclear whether the population structures of disease-causing clones differ substantially from colonizers. Studies comparing $S$. aureus strains from community carriage with those from community-acquired bacteraemias did not find a difference in diversity. ${ }^{9}$ and 10 Moreover, with the high investigation density of 1.05 samples/patient-day, it is the authors' belief that a representative sample of clinically relevant strains was collected. 
Neely ${ }^{7}$ and Neely and Maley ${ }^{8}$ used strains that had been isolated previously from patients or from the hospital environment. In order to carry out the investigation under the most authentic conditions, they used garments or materials that are frequently found on hospital wards, such as cotton, terry, blend, polyester and polyethylene, and inocula typical for environmental contaminations. ${ }^{11}$ Culture intervals were for up to 60 days and this also seems to be appropriate for hospital conditions. However, any conclusions on the correlation between survival under ambient conditions, strain frequencies and diversity must take into account the fact that that most transmission occurs via the hands of healthcare workers. Contamination of the hands with nosocomial pathogens will be much lower than the inoculum used in these studies. The average survival time on the hands will also be much shorter, even if the pathogens are not eliminated by appropriate hand hygiene, due to the bactericidal effect of fatty acids in sebum and sweat. Nonetheless, differential survival of the various pathogens may have an influence on the probability of their transmission.

\section{Experimental error}

DNA-based typing methods with the highest possible discriminatory ability were used. Gram-negative organisms were typed with two methods, random amplified polymorphic desoxyribonucleic acid and AFLP, and isolates that differed in either test were regarded as different. Gram-positive pathogens were typed by macrorestriction analysis followed by resolution using PFGE. Studies have shown a high degree of concordance between these techniques as all methods are similarly sensitive to genetic alterations that are likely to determine different clones of the investigated pathogens. Method-specific differences in the discriminatory ability would not explain the gradual difference in diversity between the various species, as illustrated in Figure 1.

\section{Correlation}

Due to the large number of isolates, this is probably the first study to provide a good estimate of the correlation between the genetic diversity of clinical isolates in a hospital setting and the ambient survival of the same important nosocomial pathogens.

The ICUs studied perform daily disinfection of the patients' environment as well as specific disinfection of surfaces if contamination is noticed. Floors are cleaned routinely and not disinfected. Handwashing compliance was $30-47 \%$ for the individual ICUs during two observation studies, leaving enough opportunity for transmission of pathogens. Finally, single items or fomites from patient care could have been swapped between patients without adequate disinfection. Plenty of transmission routes remain that could plausibly explain the observed correlation between the survival time of the various pathogens and their genetic diversity.

On the other hand, pathogens that show better survival in the environment may be more likely to be found during microbiological investigations and to survive transport to the diagnostic laboratory. Therefore, conclusions from the present findings should be drawn with caution.

The existing correlation between survival times of pathogens in the patients' environment and their genetic diversity may be taken as a reminder that the inanimate environment of ICU patients could serve as a reservoir for transmission of infection. It thus points to the value of cleaning and disinfection procedures for preventing the spread of nosocomial pathogens. However, this study should not lead to extensive untargeted disinfection procedures in the patients' environment. 
There is a need to identify and include the really crucial points for transmission in the concept of infection control. Further investigations are needed to corroborate these findings, and to identify the most important transmission routes and the most appropriate measures for infection control.

\section{Acknowledgements}

The authors thank Michael Behnke, Elke Dinger, Ulf Göbel, Elke Halle, Daniel Jonas, Ingo Klare, Reinhold Schiller, Klaus Weist and Wolfgang Witte for their substantial contribution to the transmission study.

\section{References}

1 D. Maki, C. Alvarado and C. Hassemer et al., Relation of the inanimate hospital environment to endemic nosocomial infection, N Engl J Med 307 (1982), pp. 1562-1566.

2 B. Hota, Contamination, disinfection, and cross-colonization: are hospital surfaces reservoirs for nosocomial infection?, Clin Infect Dis 39 (2004), pp. 1182-1189.

3 F. Allerberger, G. Ayliffe and M. Bassetti et al., Routine surface disinfection in health care facilities: should we do it?, Am J Infect Control 30 (2002), pp. 318-319.

4 S. Lemmen, H. Häfner and D. Zolldann et al., Distribution of multi-resistant Gram-negative versus Gram-positive bacteria in the hospital inanimate environment, J Hosp Infect 56 (2004), pp. 191-197.

5 B. Walther and P. Ewald, Pathogen survival in the external environment and the evolution of virulence, Biol Rev Camb Philos Soc 79 (2004), pp. 849-869.

$6 \mathrm{H}-\mathrm{J}$. Grundmann, S. Bärwolff and F. Schwab et al., How many infections are caused by transmission in intensive care units?, Crit Care Med 33 (2005), pp. 946-951.

7 A. Neely, A survey of Gram-negative bacteria survival on hospital fabrics and plastics, J Burn Care Rehabil 21 (2000), pp. 523-527.

8 A. Neely and M. Maley, Survival of enterococci and staphylococci on hospital fabrics and plastic, J Clin Microbiol 38 (2000), pp. 724-726.

9 H. Grundmann, S. Hori and C. Webster et al., Determining the genetic structure of the natural population of Staphylococcus aureus: a comparison of multilocus sequence typing with PFGE, RAPD and phage typing, J Clin Microbiol 40 (2002), pp. 4544-4546.

10 E. Feil, J. Cooper and H. Grundmann et al., How clonal is Staphylococcus aureus?, J Bacteriol 185 (2003), pp. 3307-3316.

$11 \mathrm{~J}$. Steer, R. Papini and A. Wilson et al., Quantitative microbiology in the managment of burn patients; I: correlation between quantitative and qualitative burn wound biopsy culture and surface alginate swab cultures, Burns 22 (1996), pp. 173-176. 